\title{
介离子化合物的合成与生物活性研究进展
}

\author{
梁伟谭成侠翁建全刘幸海* \\ (浙江工业大学化学工程学院 杭州 310014)
}

\begin{abstract}
摘要 介离子化合物代表了一类在杂环化学中非常重要的分子, 在医药和农药等领域有着广泛的应用. 自 1935 年该类 化合物被发现以来，因其有着独特的反应活性和物活性，人们对其合成、应用和性质等方面一直进行研究，不少文献报 道了该类化合物的合成及相关活性. 综述了几类介离子化合物的合成方法和部分重要机理，并探讨了介离子化合物作 为一种优势活性结构在未来的应用前景.
\end{abstract}

关键词 介离子化合物; 合成; 生物活性

\section{Advances on Synthesis and Biological Activities of Mesoionic Compounds}

\author{
Liang, Wei Tan, Chengxia Weng, Jianquan Liu, Xinghai* \\ (College of Chemical Engineering, Zhejiang University of Technology, Hangzhou 310014)
}

\begin{abstract}
As an important kind of heterocyclic molecules, mesoionic compounds are widely used in medicine, pesticide and other fields. Ever since they were discovered in 1935, mesoionic compounds have been extensively explored in terms of synthesis, properties and applications. Many references relating to these compounds have been published over years revealing their unique reactivity and bioactivity. An overview of mesoionic compounds from the perspectives of synthetic methods, reaction mechanisms and applications is offered. Their potentials as a dominant active structure for development of pesticides are also discussed.
\end{abstract}

Keywords mesoionic compound; synthesis; biological activity

1935 年, Mackney 和 Earl 合成了一种稳定的晶体化 合物, 该化合物在碱性或酸性条件下均能够水解 ${ }^{[1]}$, 并 以其发现地地名将此化合物命名为悉尼酮. 随后, Huisgen 等 ${ }^{[2]}$ 发现了慕尼黑酮, Arndtsen 等 ${ }^{[3]}$ 发现了蒙特 利尔酮，近年来杜邦公司开发了三氟苯嘧啶化合物等. 这类化合物的结构有一个共同的特点, 为六元或者五元 杂环的偶极化合物, 环内的 $\pi$ 电子处于离域的状态, 难 以用传统的价键结构进行表示 ${ }^{[4]}$, 为了区别与其他两性 离子化合物与内盐, 人们引入了介离子化合物的概念 ${ }^{[5]}$. 介离子的这种特殊环状结构, 使其有很好的反应活性, 可以发生各种亲电取代反应, 也可作为亲双烯体与烯 烃 ${ }^{[6]}$ 或炔烃 ${ }^{[7]}$ 进行 1,3 -偶极环加成反应. 此外介离子化 合物还具有良好的生物活性, 在杀虫 ${ }^{[8-10]}$ 、抗菌 ${ }^{[11-13]}$ 、抗 炎 ${ }^{[14]}$ 、抗抑郁 ${ }^{[15]}$ 和抗肿瘤 ${ }^{[16]}$ 方面有着良好的药理活性. 如杜邦公司报道了一类新型的介离子化合物 dicloro- mezotiaz 和 Triflumezopyrim，活性结果表明部分化合物 表现出一定的杀虫和抗菌活性. 因此介离子化合物在农 药和医药等方面具有广泛的市场和应用前景.

\section{1 合成及应用}

\section{1 以城市命名的介离子化合物}

\subsection{1 悉尼酮}

悉尼酮全称 1,2,3-噁二㟇-5-氧化物，是人们首次发 现的介离子类化合物. 1935 年, Mackney 和 Earl 等 ${ }^{[1]}$ 以 $N$-苯基甘氨酸作原料, 与亚硝酸反应生成 $N$-亚硝基 $-N$ 苯基甘氨酸, 然后经乙酸酕处理后得到悉尼酮(Scheme 1). 该化合物在酸性条件下水解生成肼、二氧化碳和甲 酸. 在碱性条件下加热水解生成 $N$-亚硝基氨基酸.

2004 年, Moustafa 等 ${ }^{[17]}$ 将氨基苯甲酸作为起始原 料, 依次加入氯乙酸钠、亚硝酸和醋酐后合成了 3-芳基

* Corresponding author. E-mail: xhliu@zjut.edu.cn

Received March 29, 2020; revised May 13, 2020; published online May 29, 2020.

Project supported by the Natural Science Foundation of Zhejiang Province (No. LY19C140002).

浙江省自然科学基金(No. LY19C140002)资助项目. 


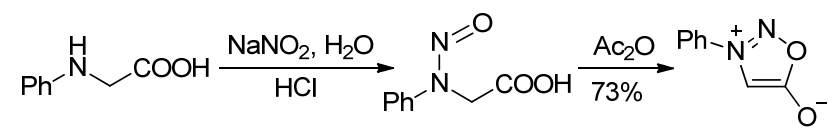

图式 1 以 $N$-苯基甘氨酸作为原料合成悉尼酮

Scheme 1 Synthesis of sydnone from $N$-phenylglycine

悉尼酮. 对 3-芳基悉尼酮的羧基基团进行修饰，与 2-氯$N$-苯基乙酰胺的氯原子发生取代反应得到 $\mathbf{I}$; 或羧基在 浓硫酸下经过酯化及胺解, 最终与醛缩合得到 II, 合成 路线如 Scheme 2. 并对所合成的化合物进行了抑菌活性 测试, 结果表明部分化合物表现出很好的抑菌活性, 特 别是化合物 III, 对测试的菌种的最低抑菌浓度 $(\mathrm{MIC}) \leqslant$ $1 \mu \mathrm{g} / \mathrm{mL}$, 与对照药剂环丙沙星比较接近, 可作为先导 化合物进一步研究.

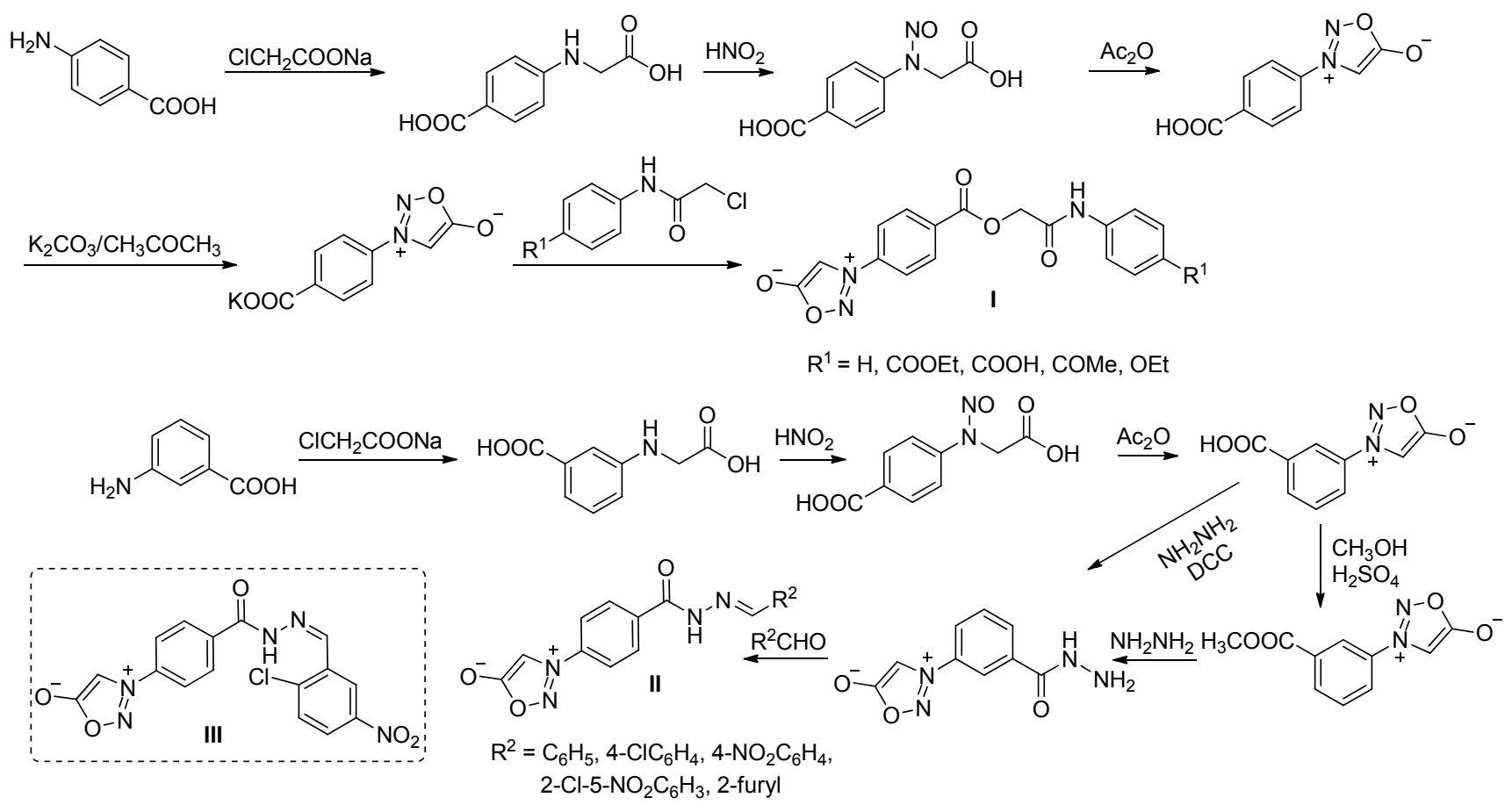

图式 2 介离子化合物 I 和 II 的合成路线

Scheme 2 Synthetic routes of mesogenic compounds I and II

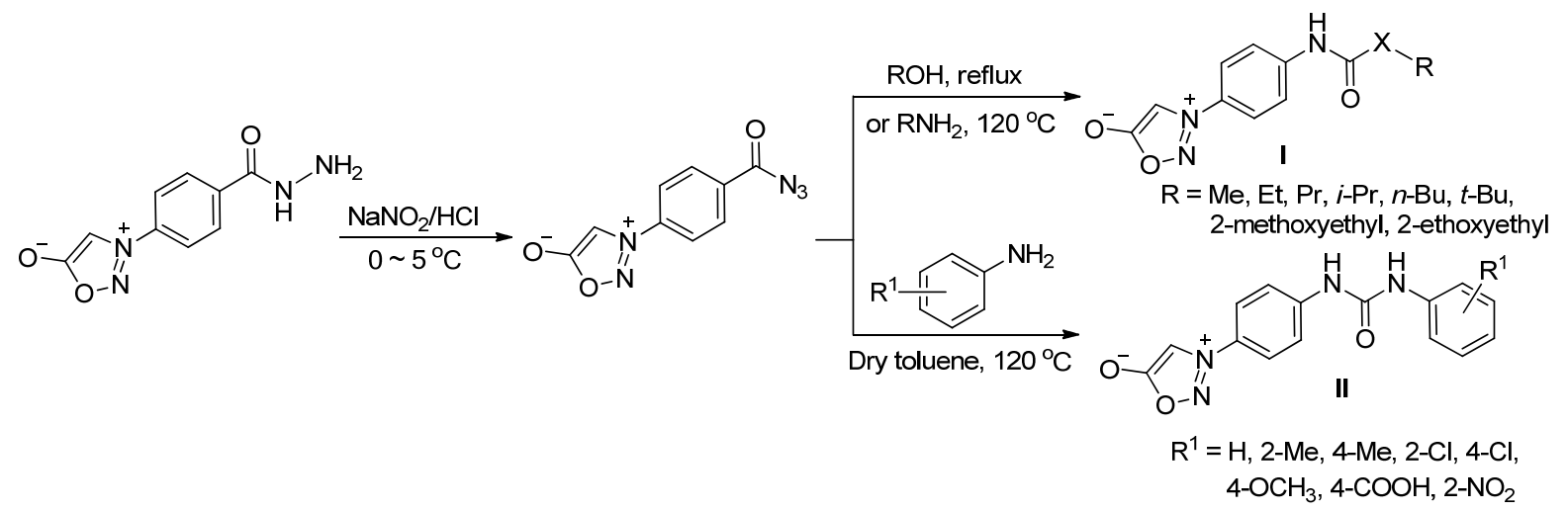

图式 3 含氨基甲酸酯或腿的悉尼酮化合物的合成路线

Scheme 3 Synthetic route of sydnone containing carbamate or urea group 
2011 年, Taj 等 ${ }^{[19]}$ 通过离子液体和四丁基溴化铵的 催化作用, 将吡唑啉衍生物改造为具有亚甲基桥链的曼 尼希碱化合物(Eq. 1). 这些化合物因含有悉尼酮这一经 典的介离子基团而具有介离子的特性. 在 $100 \mu \mathrm{g} / \mathrm{mL}$ 下 进行了相应的抗结核和抗菌活性测试, 结果表明该系列 化合物均表现出很好的抗细菌、抗真菌和抗结核活性, 其 MIC 值分别为 $0.5 \sim 4 、 1.5 \sim 3.5$ 和 $<5 \mu \mathrm{g} / \mathrm{mL}$.

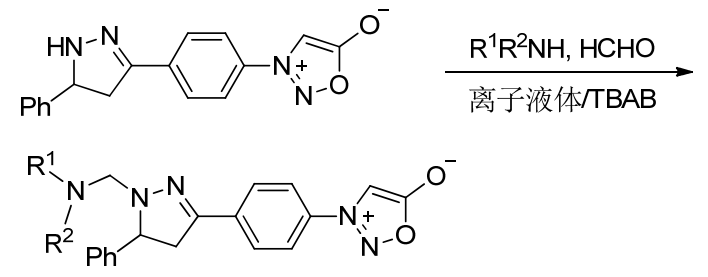

2011 年, Patel 等 ${ }^{[20]}$ 以 4-甲基-7-羟基-8-硝基香豆素 为先导化合物, 在酸性条件下通过硝化, 后与金属钠还 原引入氨基, 氨基上通过引入亚硝基与酯基得到了 $\mathrm{N}$ 亚硝基氨基酸, 在醋酸酐作用下脱水环化生成了一系列 含香豆素的悉尼酮化合物(Scheme 4). 以青霉素和氨苄 青霉素作为对照药剂进行生物活性测试, 结果显示该类 化合物抗细菌的 MIC 值在 $125 \sim 500 \mu \mathrm{g} / \mathrm{mL}$, ZI 值在 15 26 $\mu \mathrm{g} / \mathrm{mL}$ 之间, 整体活性低于对照药剂. 对大肠杆 菌、假单胞杆菌、金黄色酿脓葡萄球菌和枯草芽孢杆菌 具有中等的抑制活性.<smiles>Cc1cc(=O)oc2cc(O)ccc12</smiles>

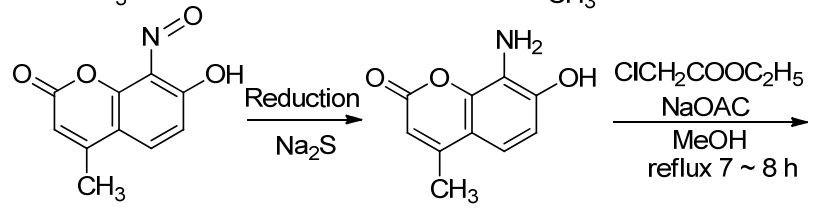<smiles>CCOC(=O)CNc1c(O)ccc2c(C)cc(=O)oc12</smiles>

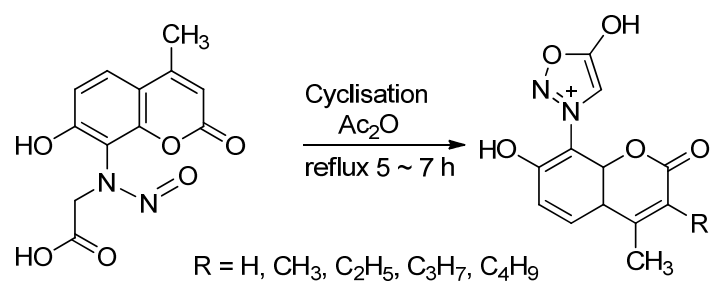

图式 4 含香豆素的悉尼酮化合物的合成

Scheme 4 Synthesis of sydnone containing coumarin moiety
2013 年, Hegde 等 ${ }^{[21]}$ 以 1,2-二氨基乙烷和 2,3-二溴1-(3-芳基悉尼酮-4-基)-3-芳基丙烷基-1-酮作为原料, 1,2-二氨基乙烷与羰基脱水缩合后，进行两次亲核取代 后形成一个含氮三元环的衍生物, 合成路线如 Scheme 5 所示. 以环丙沙星和环吡酮胺作为对照药剂, 并以真菌 和细菌为研究对象, 采用杯碟法进行抑菌活性测试. 结 果显示, 当 $\mathrm{R}=\mathrm{H}, \mathrm{Ar}=3,4$-亚甲基二氧苯基时, 对金黄 色葡萄球菌、肺炎克雷伯菌、黑曲霉和白色念珠菌抑制 直径分别为 $18,22,26,26 \mathrm{~mm}$. 与对照药剂活性相当, 显示出良好的杀菌活性.

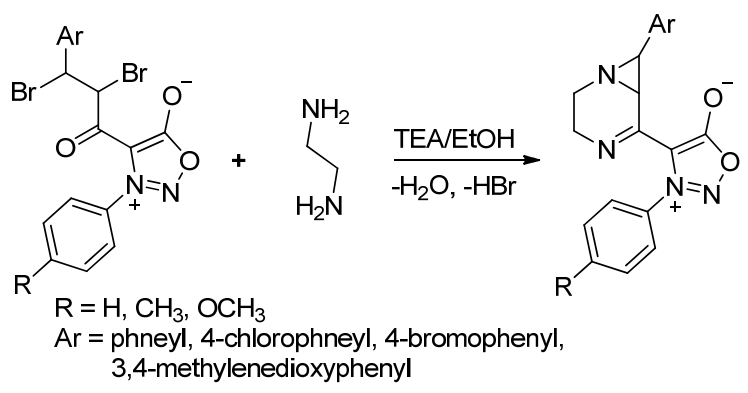

图式 5 含氮杂环丙烷的悉尼酮化合物的合成

Scheme 5 Synthesis of sydnone containing azapropane

2014 年, Dubey 等 ${ }^{[22]}$ 对悉尼酮 2 位和 3 位进行了修 饰, 以吲哚并噻嗪作为原料合成的 $N$-亚硝基氨基酸, 在 醋酸酎作用下脱水环化生成悉尼酮，悉尼酮的 2 位在 $\mathrm{P}_{2} \mathrm{O}_{5}$ 的作用下与氯代硫酸偶联, 后被含氮的六元杂环取 代, 合成了含有吲哚并噻嗪的悉尼酮类化合物(Scheme 6). 筤选了目标化合物的抗菌、杀虫和驱虫等多种生物 活性，总体而言，这些化合物都具有较为广谱的生物活 性, 当 $\mathrm{X}=\mathrm{O}$ 时活性最好, 生物活性广谱, 其抗菌、杀虫 和驱虫活性都很好.
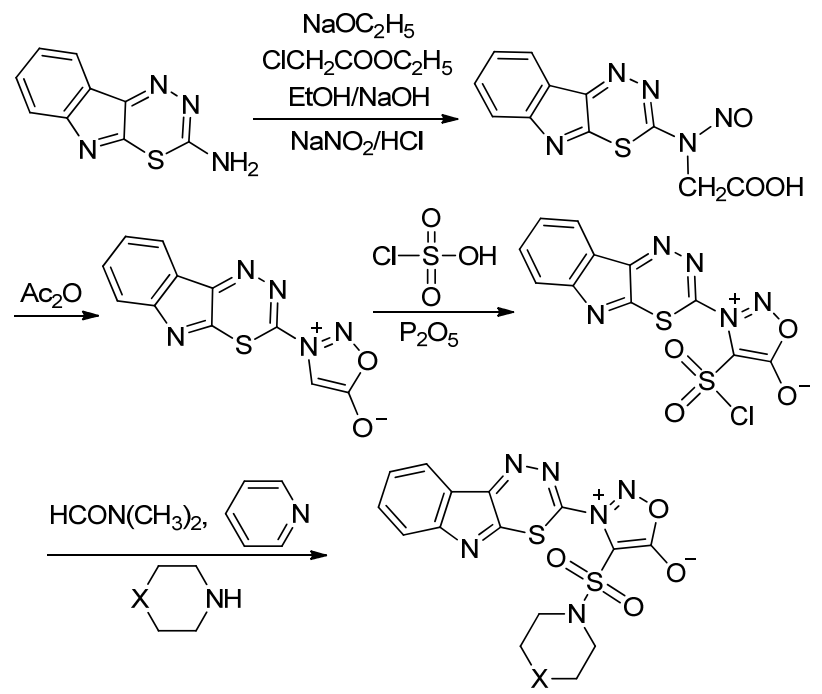

图式 6 含吲哚并噻嗪的悉尼酮类化合物的合成 Scheme 6 Synthesis of sydnone containing indolothiazine 


\section{1 .2 慕尼，黑酮}

慕尼黑酮全称为 1,3-噁唑-5-氧化物. 它是一种介离 子类化合物, 且存在一系列的共振杂化体(Scheme 7).

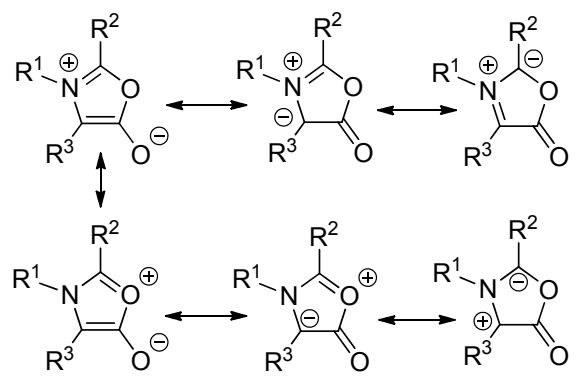

$R^{1}=$ alkyl, aryl, acyl, etc.; $R^{2}, R^{3}=H$, alkyl, aryl, etc.

图式 7 慕尼黑酮的共振式

Scheme 7 Resonance of Munichone

1970 年, Bayer 等 ${ }^{[23]}$ 利用 $N$-酰基氨基酸为原料, 在 乙酸酐脱水剂的作用下合成了慕尼黑酮(Eq. 2). 之后人 们改进了其合成方法, 用二环己基碳二亚胺(DDC)或 1乙基-(3-二甲基氨基丙基)碳酰二亚胺(EDC)替换乙酸

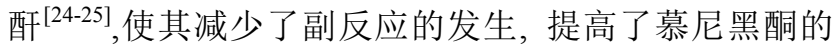
产率. 由于慕尼黑酮易水解和氧化, 所以该反应需在无 氧无水的体系中发生 ${ }^{[26]}$.

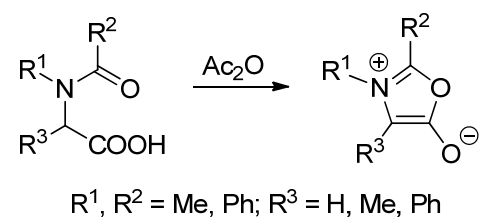

除了传统方法制备慕尼黑酮外, 近年来利用金属催 化剂合成慕尼黑酮成为了一种新途径. 2008 年, Arndtsen 等 ${ }^{[27]}$ 报道将 $\alpha$-酰胺基醚作为原料, 在 $\mathrm{CO}$ 中, 以乙腈为 溶剂, 钯作催化剂, 在 $65{ }^{\circ} \mathrm{C}$ 下发生 $\mathrm{C}-\mathrm{O}$ 键的活化和插 入一氧化碳的反应, 得到了慕尼黑酮(Eq. 3). 该反应条 件温和, 在不使用酸催化剂的情况下, 金属钯催化剂可 以直接高效地活化 $\alpha$-氨基醚中的 $\mathrm{C}-\mathrm{O}$ 键, 进而提高反 应效率和产率. 考虑到慕尼黑酮可以与一系列不饱和化 合物进行环加成, 因此该反应也为其他类型杂环化合物 的合成提供了可能的途径.

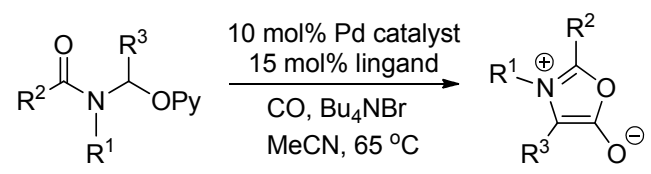

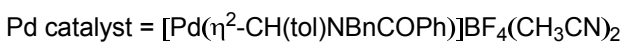
lingand $=t-\mathrm{Bu}_{2} \mathrm{P}(2-$ biphenyl) $\mathrm{R}^{1}=\mathrm{Bn}, i-\mathrm{Pr}, \mathrm{Et}, 4-\mathrm{MeOC}_{6} \mathrm{H}_{4}$ $\mathrm{R}^{2}=p$-Tol, Me, 4- $\mathrm{ClC}_{6} \mathrm{H}_{4}, 2$-furanyl $\mathrm{R}^{3}=p-\mathrm{Tol}, \mathrm{Me}, 4-\mathrm{ClC}_{6} \mathrm{H}_{4}$
机理如 Scheme 8 所示, $\alpha$-酰胺基醚的 $\mathrm{C}-\mathrm{O}$ 键在金 属钯的作用下被活化导致烷氧基团离去，羰基与金属钯 配位形成中间体 $\mathbf{I}$, 随后中间体 $\mathbf{I}$ 上的配体经过解离、钯 重新与 $\mathrm{CO}$ 配位后得到 II, 由于 II 的不稳定导致 CO 解 离并迅速迁移插入到 $\mathrm{C}-\mathrm{Pd}$ 键之间形成较稳定的六元 环配合物 III，最终在碱的作用下配合物 III 经还原消除 和脱去一分子卤化氢得到了慕尼黑酮.

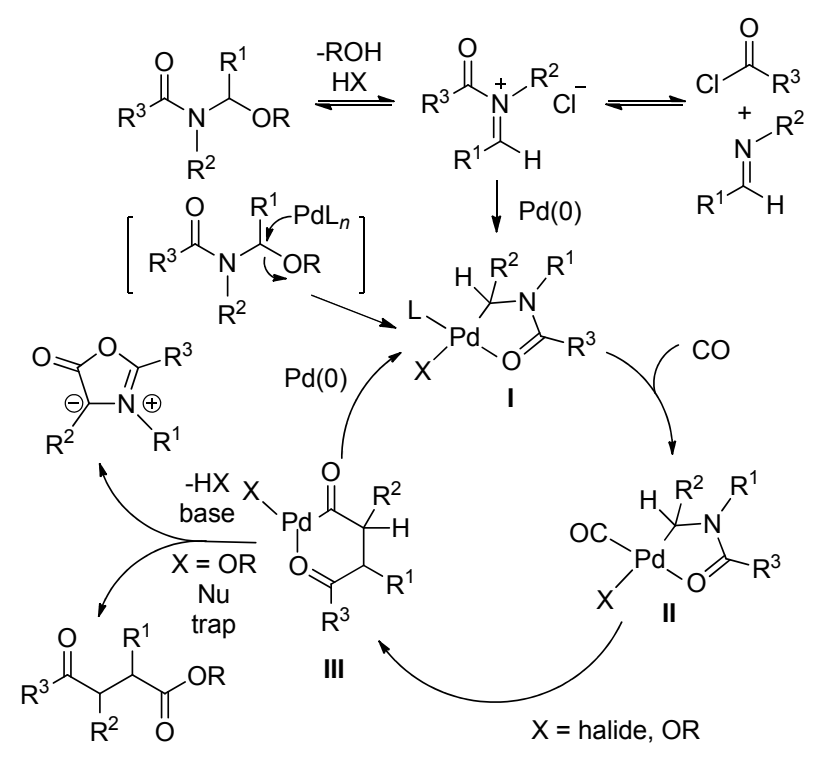

图式 8 钯催化慕尼黑酮的反应机理

Scheme 8 Reaction mechanism of Pd-catalyzed Munichone

2009 年, Arndtsen 等 ${ }^{[28]}$ 报道将亚胺和酰氯作为原料, 在 $\mathrm{CO}$ 中, 以四氢呋喃(THF)为溶剂, 钯作催化剂, 在 $55{ }^{\circ} \mathrm{C}$ 下反应得了慕尼黑酮(Scheme 9). 相对于传统方 法利用 $N$-酰基氨基酸脱水制备慕尼黑酮，此方法提供 了合成这一类化合物的新途径，实用性较强. 显著优势 在于原料简单易得，产物的稳定性也较好，此方案可被 推广制备一系列的慕尼黑酮.

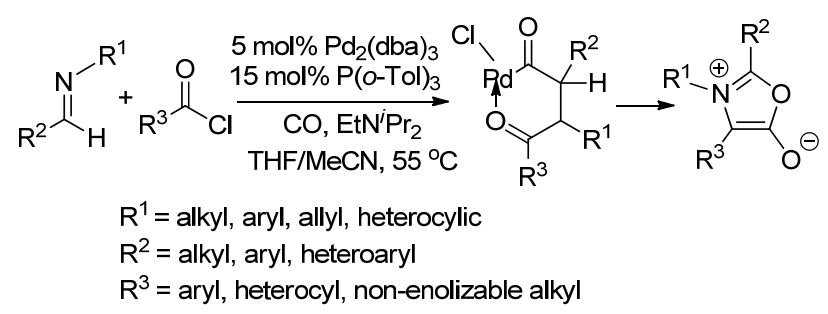

图式 9 慕尼黑酮的新合成方法

Scheme 9 New synthetic method of Munichone

该反应催化可能的机理如 Scheme 10 所示, 亚胺与 酰氯通过亲核取代形成酰胺正离子，活化零价钯与其配 位后得到中间体 $\mathbf{I}$ ，中间体 $\mathbf{I}$ 原有的配体进行解离后与 $\mathrm{CO}$ 和 $\mathrm{Cl}^{-}$再配位形成中间体 II，由于中间体 II 的不稳 定性，中间体 II 上的 $\mathrm{CO}$ 配体解离后迁移插入到 C-Pd 
键生成中间体 III, 最终中间体 III 消除一分子盐酸形成 的 IV 经脱去钯、环化形成 C-O 键后合成了目标产物 慕尼黑酮, 其中 $\mathrm{CO}$ 作为碳源引入增加了一个碳, 且通 过催化构建了 $\mathrm{C}-\mathrm{C}$ 键在反应中起了重要作用.

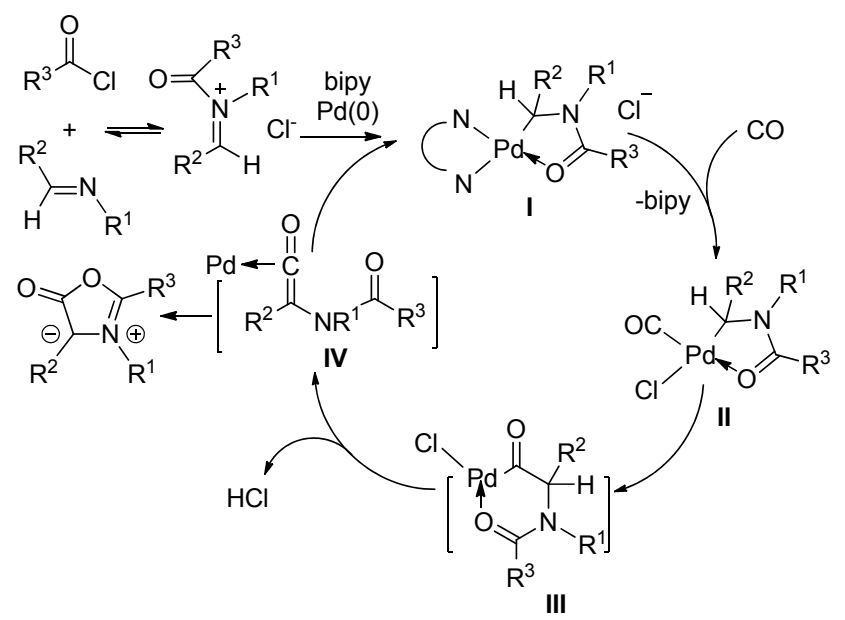

图式 10 钯催化 $\alpha$-酰胺反应的机理

Scheme 10 Mechanism of Pd-catalyzed $\alpha$-amide reaction

2015 年, 苗群等 ${ }^{[29-30]}$ 为了证实慕尼黑酮的生成终 止多肽合成这一路径, 设计了多肽端基模拟物, 实验结 果表明该化合物易发生 $\beta$ 消除, 很不稳定, 脱掉 $\mathrm{HCo}(\mathrm{CO})_{4}$ 后生成了慕尼黑酮(Scheme 11).

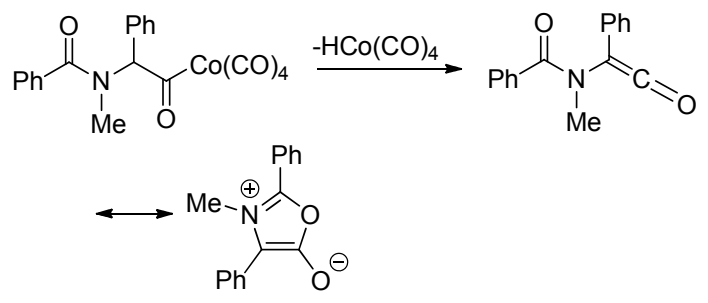

图式 11 酰基钴化合物消去钴酸生成慕尼黑酮

Scheme 11 Münchnones formation from acylcobalt compounds by elimination of $\mathrm{HCo}(\mathrm{CO})_{4}$

2016 年, Arndtsen 等 ${ }^{[31]}$ 报道了在乙腈中, 将亚胺、对 甲碘苯和 $\mathrm{CO}$ 作为原料, 活化金属钯作为催化剂, $55{ }^{\circ} \mathrm{C}$ 加热反应 $16 \mathrm{~h}$, 合成了慕尼黑酮(Eq. 4). 该反应中, 活化 金属钯与芳基碘的氧化加成、 $\mathrm{CO}$ 的插入都是迅速进行的, 生成了易于分离、产率高的络合物 $\mathbf{I}$, 且在碱 $\mathrm{NEt}^{i} \mathrm{Pr}_{2}$ 的作 用下, 可抑制络合物 $\mathbf{I}$ 与亚胺、 $\mathrm{CO}$ 生成味唑啉羧酸盐副 产物，从而提高了目标产物产率，并对其反应机理作了 相应解释(Scheme 12), 活化的零价金属钯通过与芳卤 代烃进行氧化加成、羰基插入后生成钯芳基配合物 $\mathbf{I}$, 随 后中间体 $\mathbf{I}$ 与不饱和亚胺的双键进行插入反应、钯与羰 基配位后形成中间体 II, 由于 II 的不稳定性, 导致可以 迅速地与羰基缔和并插入, 最终经过配体解离还原消除 反应和脱去质子后即可得慕尼黑酮 III.
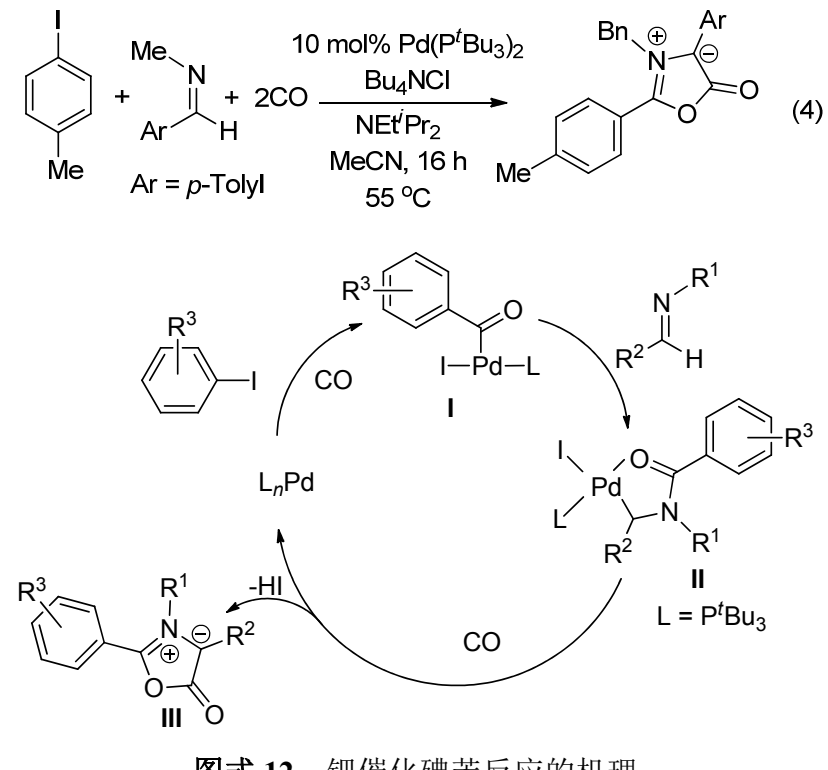

Scheme 12 Mechanism of Pd-catalyzed iodobenzene reaction

\subsection{3 蒙特利尔酮}

近年来人们又发现了一种新的 1.3-偶极体, 其一系 列共振杂化体(Scheme 13)与慕尼黑酮有所区别, 五元 环中含有磷, 因此称之为磷杂慕尼黑酮或蒙特利尔酮.

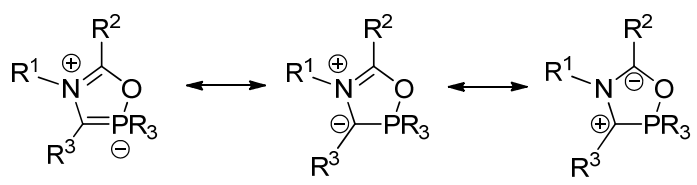

图式 13 蒙特利尔酮的共振式

Scheme 13 Resonance of Montrealone

2007 年, Arndtsen 等 ${ }^{[32]}$ 首次报道了将亚胺、酰氯作 为原料反应制得亚胺盐，再与三价磷化合物进一步反应 得到磷盐, 磷盐在 1,5-二氮杂二环[5.4.0]十一烯-5 (DBU)作用下脱氢生成了蒙特利尔酮(Scheme 14). 从蒙

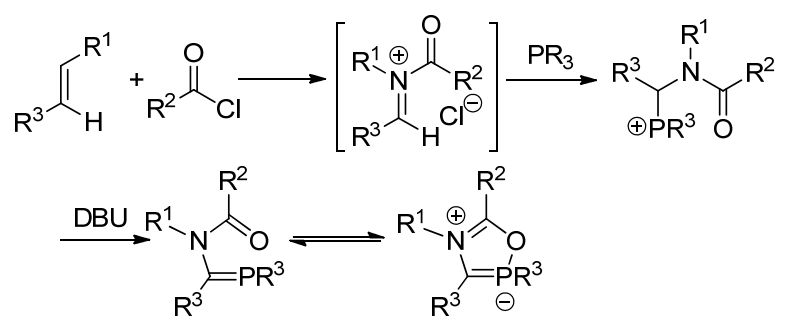

$\mathrm{R}^{1}=$ alkyl, aryl, allyl, heterocylic; $\mathrm{R}^{2}=$ aryl, heterocylic, $i-\mathrm{Pr}, t-\mathrm{Bu}$; $\mathrm{R}^{2}=$ aryl, heterocylic, $i-\mathrm{Pr}, t-\mathrm{Bu}$;

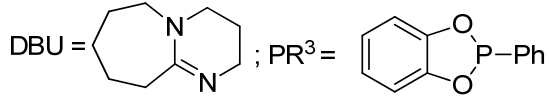

图式 14 以亚胺、酰氯为原料合成蒙特利尔酮 Scheme 14 Synthesis of montreal ketone from imine and acid chloride 
特利尔酮的结构上看, 它相当于以一个三取代膦基团 $\mathrm{PR}^{3}$ 替代了慕尼黑酮中原有的羰基.

Couture 等 ${ }^{[33]}$ 将三价磷化物、亚胺和酰氯作为原料, 在二氯甲烷溶剂中得到了酰胺基取代的 HornerWadsworth-Emmons 试剂. 2010 年, Arndtsen 等 ${ }^{[34]}$ 对反应 物做了进一步的研究, 他们用 Lewis 酸处理该反应物, 结果却合成了蒙特利尔酮(Scheme 15).

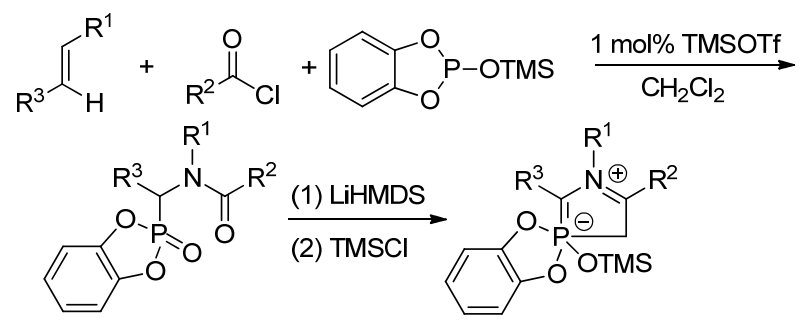

图式 15 蒙特利尔酮的新合成方法

Scheme 15 Thenew methodofm ontr6alonessynthesis

2013 年, 该课题组 ${ }^{[35]}$ 又发现了蒙特利尔酮的新合 成方法, 将含有碳一碳双键的亚胺和酰氯作为原料, 加 入三取代磷化合物和 DBU 制备了蒙特利尔酮(Eq. 5).

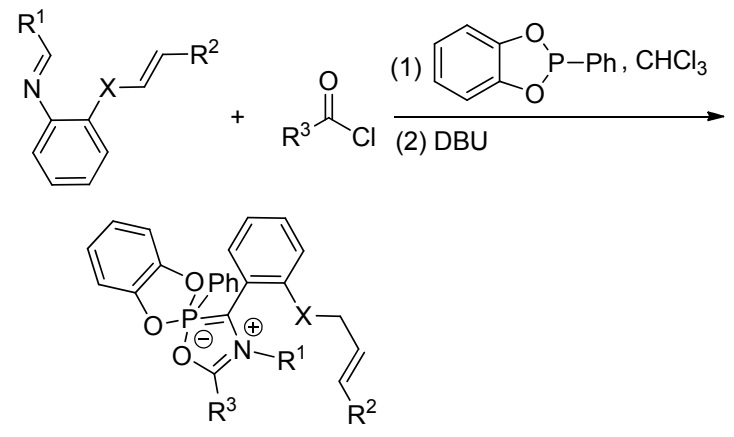

\section{2 吡啶并嘧啶类介离子化合物}

嘧啶酮类介离子化合物在防治害虫方面显示出优 异的活性, 引发了人们进一步探索, 下文简单介绍几种 合成路径 ${ }^{[36-37]}$ (Scheme 16). 路线 1, 将取代 1,3-丙二酮 和取代二甲胺作为原料合成目标物, 该方法也是合成该 化合物的通用路线. 路线 2 , 利用钯催化, 与卤代的介 离子化合物发生 $\mathrm{C}-\mathrm{C}$ 键偶联, 得到该化合物, 此方法 原料价格高且反应条件苛刻. 路线 3, 将取代二甲胺和 $\mathrm{C}_{3} \mathrm{O}_{2}$ 作为原料合成介离子母环, 然后对母环 3 位进行修 饰, 利用铜催化, 使之与活泼卤化物进行 $\mathrm{C}-\mathrm{C}$ 键偶联 制得.

2014 年, Lugan 等 ${ }^{[38]}$ 以 2-溴苯甲酸为原料, 利用氯 化亚砜对羧酸官能团氯化得到酰氯, 在三乙胺溶剂中, 异丙基胺对其亲核加成生成中间体 I, 中间体 I 与异丙 基胺缩合合成亚氨基苯乙胺关键中间体 II, 最终经过中 间体 II 与 2-甲基丙二酰氯进行分子内的环化, 合成了甜 菜碱嘧啶(Scheme 17). 此方案反应快速且操作简单.

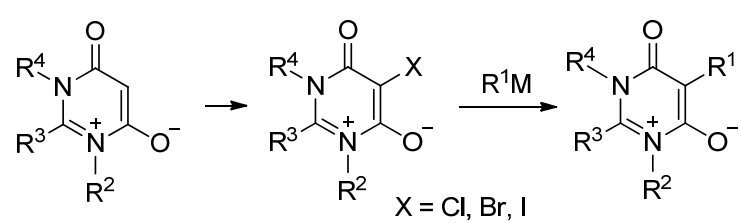

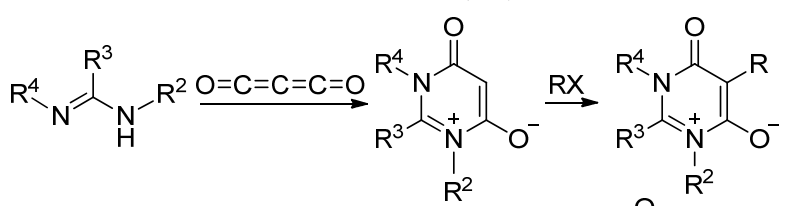

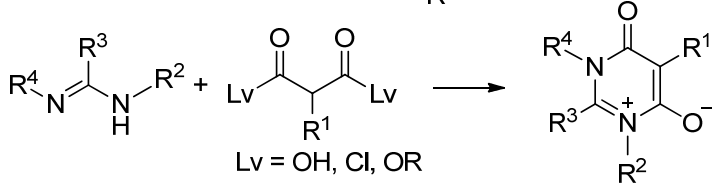

图式 16 嘧啶酮类介离子化合物的几种合成路径 Scheme 16 Several synthetic routes of pyrimidinone-type mesoionic compounds

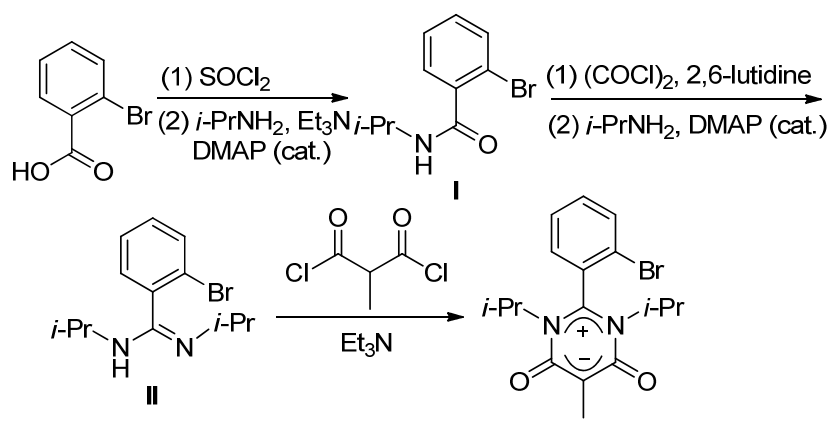

图式 17 甜菜碱嘧啶的合成

Scheme 17 Synthesis of betaine pyrimidine

2017 年, 张文明等 ${ }^{[39]}$ 等将 2-氨基吡啶作为原料, 然 后与 5-醛基嘧啶缩合, 后经 $\mathrm{NaBH}_{4}$ 还原得到中间体 $N$-(5-嘧啶基)甲基-2-吡啶胺 II, 该操作繁琐. 其次在制 备中间体 2-[3-(三氟甲基)苯基]丙二酸酯 I 后与 II 环化, 得到三氟苯嘧啶(Scheme 18). 在合成 I 的过程中, 反应 条件较为苛刻, 而且三氯苯酚对环境有很大污染.

2017 年, 英伍君等 ${ }^{[0]}$ 以 5-酫基嘧啶和 2-氨基吡啶 为原料一步合成 $N$-(5-嘧啶基)甲基-2-吡啶胺(I)，操作简 便. 将间三氟甲基苯乙酸作为原料制备 2-[3-(三氟甲基) 苯基]丙二酸二甲酯(II). 相对于偶联反应需在无水无氧 的体系中, 该合成路线操作简单, 原料易得, 条件温和. 其次合成 2-[3-(三氟甲基)苯基]丙二酸后没有引入大的 离去基团三氯苯酚, 缩短了反应步骤(Scheme 19).

2017 年, 张文明等 ${ }^{[4]}$ 将 2-氨基-3-甲基吡啶作为原 料，与 2-氯-5-氯甲基噻唑发生亲核取代反应制得中间 体 II. 该过程中芳胺经保护基保护、卤代烷取代、去保 护基三步制得仲胺中间体 II. 该路线原料易得，适合多 种结构类型反应物. 以 1,3-二氯-5-碘苯为原料, 在 $\mathrm{Cs}_{2} \mathrm{CO}_{3}$ 作用下与丙二酸甲酯偶联, 经过 $\mathrm{NaOH}$ 水解得到 中间体 I. 相对于含活性亚甲基氧基取代物在碱的作用 


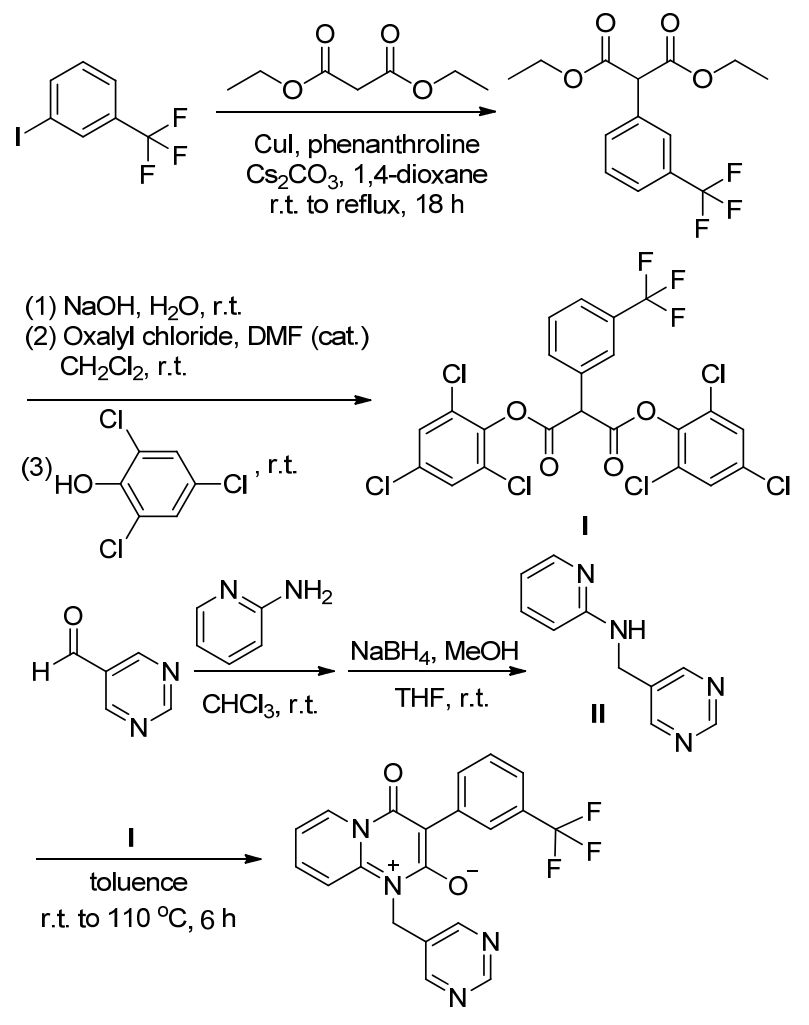

图式 18 三氟苯嘧啶的合成路线

Scheme 18 Synthesis route of triflumezopyrim<smiles>CC(C)(C)[C@H](O)Cc1cccc(NCc2cncnc2)n1</smiles><smiles>COC(=O)Cc1cccc(C(F)(F)F)c1</smiles>

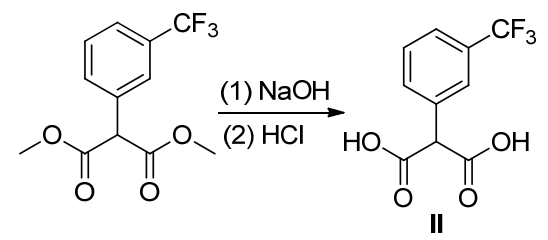

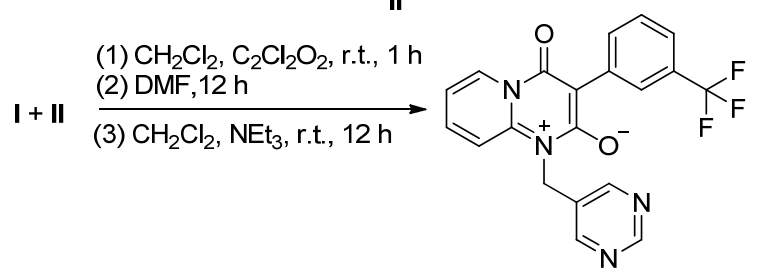

图式 19 改进后 Triflumezopyrim 的合成

Scheme 19 Improved synthesis method of triflumezopyrim 下与酸二酯衍生物缩合、酸化、酯化制得取代丙二酸二 酯中间体. 此路径步骤简便且反应收率较高. 最后中间 体 II 和 I 在有机溶剂中加热回流即可得到 Dicloro- mezotiaz (Scheme 20).<smiles>COC(=O)CC(=O)OC</smiles><smiles>COC(=O)C(C(=O)OC)c1cc(Cl)cc(Cl)c1</smiles>

(1) $\mathrm{NaOH}, \mathrm{H}_{2} \mathrm{O}, \mathrm{MeOH}$, r.t. $\sim 10{ }^{\circ} \mathrm{C}$

(2) $\mathrm{HCl}, \mathrm{H}_{2} \mathrm{O}$<smiles>O=C(O)C(C(=O)O)c1cc(Cl)c(I)c(Cl)c1</smiles><smiles>CC(=O)Nc1ncccc1C</smiles>
(2) $\mathrm{NaOH}, 50^{\circ} \mathrm{C}$ (1) $\mathrm{K}_{2} \mathrm{CO}_{3}, n-\mathrm{Bu}_{4} \mathrm{NBr}$ (cat.)

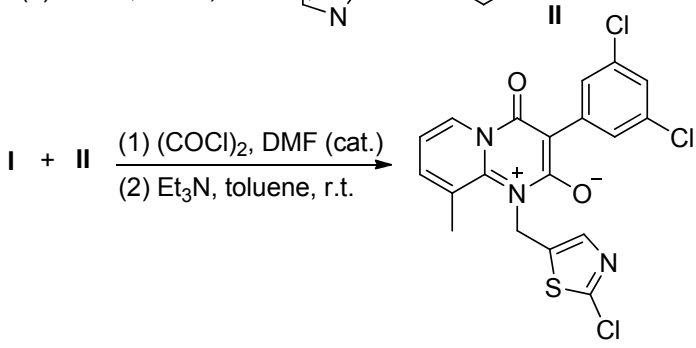

图式 20 Dicloromezotiaz 的合成路线

Scheme 20 Dicloromezotiaz's synthetic route

2019 年，宋宝安等 ${ }^{[42]}$ 将香豆素衍生物的部分片段 引入到吡啶并 $[1,2-\alpha]$ 嘧啶酮的骨架中, 构建了一系列新 颖的吡啶并 $[1,2-\alpha$ 嘧啶酮类介离子化合物. 目标化合物 制备过程如 Scheme 21 所示. 生物活性测试结果显示该 化合物具有良好的抗菌活性. 当 $\mathrm{R}^{1}=\mathrm{OCH}_{3}, \mathrm{R}^{2}=4-\mathrm{CH}_{3}-$ $\mathrm{C}_{6} \mathrm{H}_{4} \mathrm{CH}_{2}$ 时, 对水稻白叶枯病菌表现出优异的体外活 性, 其 $\mathrm{EC}_{50}=1.1 \mu \mathrm{g} / \mathrm{mL}$, 优于对照药叶枯唑 $(92.7 \mu \mathrm{g} /$ $\mathrm{mL}$ )和噻菌铜 $(105.4 \mu \mathrm{g} / \mathrm{mL})$. 在 $200 \mu \mathrm{g} / \mathrm{mL}$ 活体测试下, 其对水稻枯萎病的保护和治疗活性分别达 $75.12 \%$ 和 $72.04 \%$, 高于叶枯唑(62.24\%和 50.83\%)、噻菌铜(53.35\% 和 65.04), 为其作为新型抗菌剂的应用提供了基础.<smiles>[R]Oc1ccc(Cn2c(=O)c(C)c([O-])[n+]3ccccc23)cc1[R]</smiles>

图式 21 吡啶并[1,2- $a$ ] 嘧啶酮类介离子化合物的合成路线 Scheme 21 Synthesis of pyrido[1,2-a]pyrimidone mesoionics 
2016 年, 日本化药株式社会将氰基乙基引入到吡 啶并 $[1,2-\alpha]$ 嘧啶酮类介离子化合物核心环的 1 位 ${ }^{[43]}$. 当 浓度为 $0.1 \mu \mathrm{g} / \mathrm{mL}$ 时, 该类化合物对棉蚜致死率接近 $100 \%$ ，生物活性优于杜邦公司开发的 Triflumezopyrim (浓度为 $0.39 \mu \mathrm{g} / \mathrm{mL}$ 时对棉蚜致死率约 60\%) 和 Dicloromezotiaz(浓度为 $50 \mu \mathrm{g} / \mathrm{mL}$ 时对棉蚜致死率约 $90 \%$ ).<smiles>N#CCC[n+]1c([O-])c(-c2cccc(C(F)(F)F)c2)c(=O)n2ccccc21</smiles>

\section{3 其它唑类介离子化合物}

Thieme 等 ${ }^{[44]}$ 通过比较 3-甲基-2-苯基-1,3,4-噻二唑5-硫醇及其相对应的非内盐型同分异构体中硫、氮结合 能差异, 确认前者为介离子化合物. 2000 年, 张自义 等 ${ }^{[45}$ 对该化合物其进行了改造, 使其 2 位含有芳胺基, 3 位不含取代基. 在乙醇溶剂中, 利用 $\omega$-溴代- $\omega$ - $(1 H-$ 1,2,4-三唑)苯乙酮与 2-芳胺基-1,3,4-噻二唑-5-硫醇反应 生成了稳定的噻二唑介离子化合物(Scheme 22).

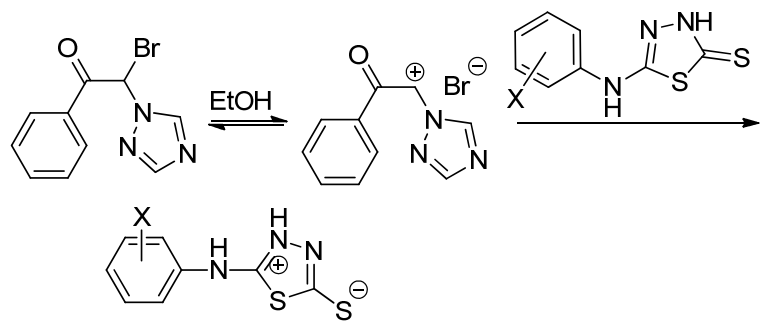

图式 22 噻二唑介离子化合物的合成

Scheme 22 Synthesis of thiadiazole mesogenic compounds

2002 年, Abbott 等 ${ }^{[46]}$ 利用盐酸、亚硝酸钠和芳胺反 应生成重氮盐, 将 4-氧-1,2,3,4-四氢喹唑啉-2-羧酸作为 起始原料, 在吡啶或酸䣶溶剂的作用下, 与重氮盐进行 分子间的环化生成中间体 $\mathbf{I}$, 中间体 $\mathbf{I}$ 与三氯氧磷加热 回流 $2 \mathrm{~h}$, 进一步与嗍氢化钠或格氏试剂等反应得到了 介离子化合物 III (Scheme 23).

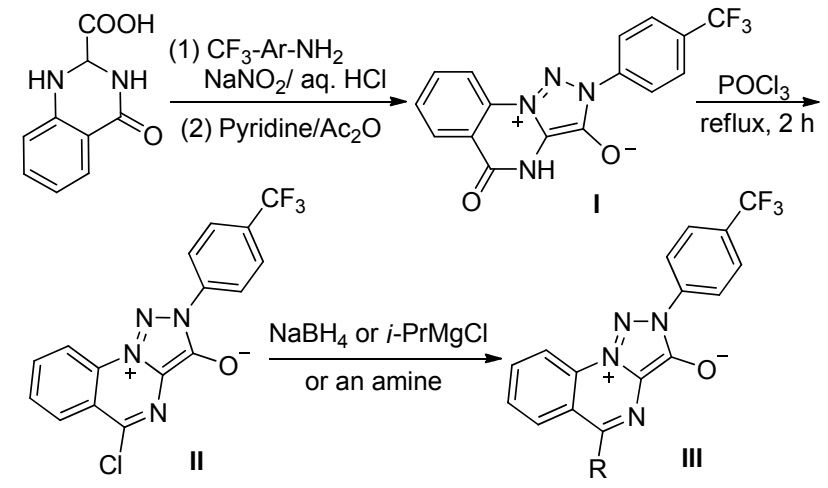

图式 23 异喹啉衍生物的合成

Scheme 23 Synthesis of mesogenic isoquinoline derivatives
Avalos 等 ${ }^{[47-49]}$ 报道了以 $N, N, N^{\prime}$-三取代硫脲和溴苯 乙酸为起始原料, 在三乙胺作用下, 发生亲核取代生成 中间体 I，在酸酐或者三乙胺溶剂中，进一步发生分子 内环化脱水合成介离子化合 II (Scheme 24). 也可用 2氯-2-苯基乙酰氯替代澳苯乙酸, 在三乙胺的作用下直 接与之发生分子间的环化合成噻唑介离子化合物，此方 法较为简便高效.

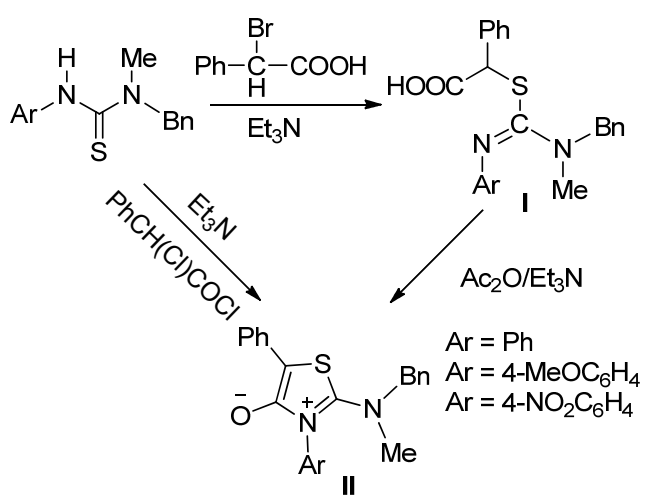

图式 24 噻唑介离子化合物的合成

Scheme 24 Synthesis of thiazole mesogenic derivatives

另外一种噻唑介离子化合物的合成路径(Scheme 25)是在二氯甲烷溶剂中，硫酰胺上硫的孤对电子可进 攻 2-氯-2-苯基乙酰氯上的氯原子, 进行亲核取代生成 中间体 III，由于中间体 III 中酰氯上羰基的活性很高, 很容易发生分子内的亲核加成消除反应，环化生成噻唑 介离子化合物 IV.
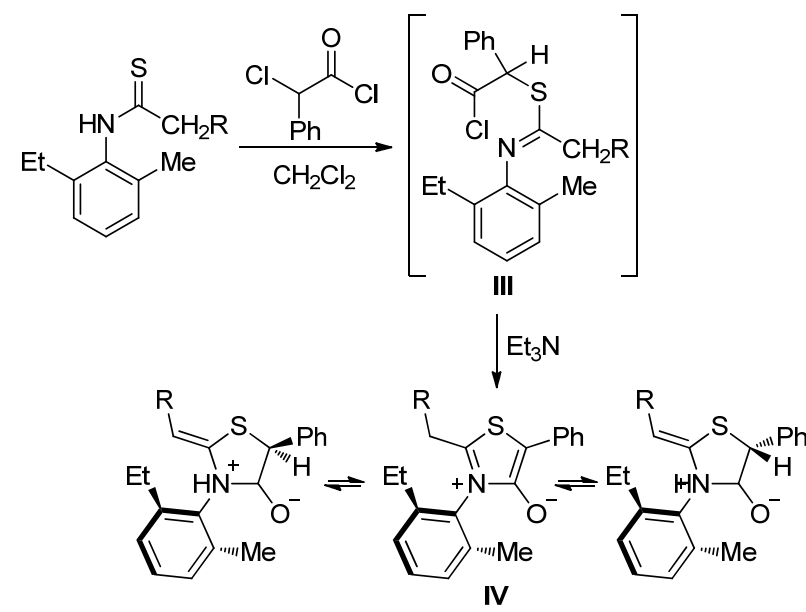

图式 25 噻唑介离子化合物 IV 的合成

Scheme 25 Synthesis of thiazole mesogenic compound IV

2010 年, Chernyshev 等 ${ }^{[50]}$ 报道以二氨基三唑和 $\beta$-酮 酸酯为原料, 在乙醇或四氢呋喃溶剂中, $145 \sim 150{ }^{\circ} \mathrm{C}$ 下 反应 $5 \mathrm{~h}$ 左右，二氨基三坐上的氨基与 $\beta$-酩酸酯的羰基 亲核加成，缩合生成了不稳定的氨基丙烯酸酯关键中间 
体, 中间体进一步发生分子内的快速环化, 最终生成三 唑并嘧啶介离子化合物(Scheme 26).<smiles>[R]C(=O)C([R])C(=O)O[AlH2]</smiles><smiles>[R]C(Nc1nc(N)n([R])n1)=C([R])C(=O)O[AlH]</smiles>

图式 26 异喹啉衍生物的合成

Scheme 26 Synthesis of mesogenic isoquinoline derivatives

2014 年, Hassan 等 ${ }^{[51]}$ 将 $N$-取代的苯基肼基碳硫代 酰胺和乙烯-1,1,2,2-四腈(TCNE)作为原料, 在乙醇溶剂 中, 经过多步的对双键的亲核加成和消除反应等过程后 得到了 1,2,4-三唑介离子化合物(Eq. 6), 当 $\mathrm{R}=\mathrm{CH}_{2} \mathrm{CH}_{3}$, 反应活性较好, 产率较高(Scheme 27), 该反应 TCNE 分 子结构中因连有 4 个强吸电子基团 $\mathrm{CN}$, 所以具有很高 的反应活性，易受亲核试剂的进攻，使得该反应能够顺 利进行.

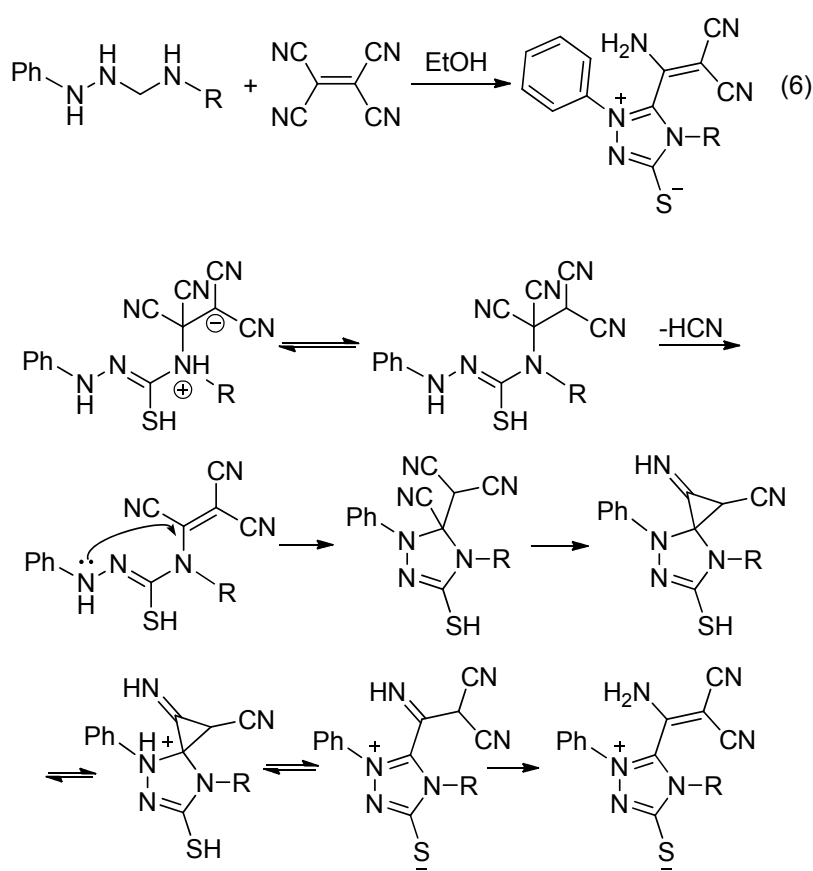

图式 27 三唑介离子衍生物的合成

Scheme 27 Synthesis of triazole mesogenic derivative

吴华悦等 ${ }^{[52]}$ 将炔烃和三唑作为原料, 在过氧化钠 或过氧化二叔丁基的作用下，利用金属铑催化剂催化氧 化, 使用一锅法, 加热 $100{ }^{\circ} \mathrm{C}$ 下持续反应 $14 \mathrm{~h}$, 定向地 活化三唑和苯环中的 $\mathrm{C}-\mathrm{H}$ 键后, 实现了 $\mathrm{C}-\mathrm{C}$ 键、 $\mathrm{C}-$ $\mathrm{N}$ 键及 $\mathrm{C}-\mathrm{O}$ 键的偶联. 环化生成异喹啉介离子衍生物 (Eq. 7). 当 $\mathrm{R}^{3} 、 \mathrm{R}^{1}=\mathrm{Me}, \mathrm{R}^{2}=\mathrm{oct}, \mathrm{R}^{4}=\mathrm{Ph}$ 时, 产率相对 较高. 该方案起始原料和催化剂易得, 且为介离子异喹
诺酮衍生物的合成提供了简便实用的方法.

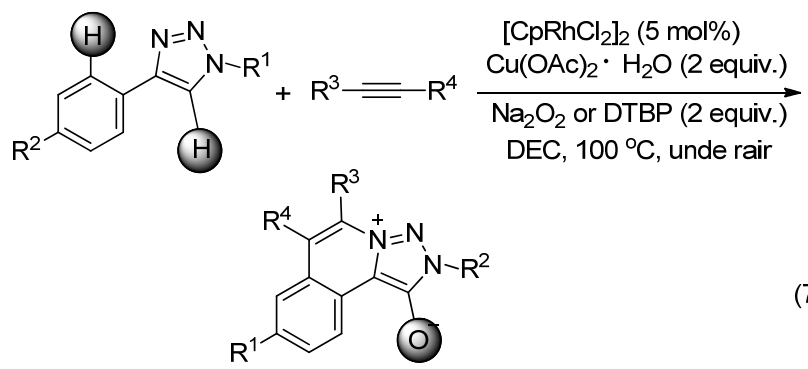

Huseyin 等 ${ }^{[53]}$ 以吡啶酰氯和 $(E)-N$-乙基-1-(对甲苯 基)甲亚胺为原料, 在乙胺和三氯乙烷有机溶剂中 $80{ }^{\circ} \mathrm{C}$ 反应约 $12 \mathrm{~h}$ 后，得到了 2-乙基-3-(对甲苯基)- $2 H$-咪唑并 [1,5- $\alpha$ 吡啶-4-1-酸(Scheme 28). 由于吡啶酰氯中羰基的 活性很高, 该过程中亚胺和酰氯加成消除生成亚胺正离 子中间体，中间体的吡啶环上的氮原子发生分子内的亲 核加成，环化后可脱去一分子盐酸，以较高的产率得到 目标产物. 该方法反应活性好, 无其它副反应发生, 在 无需过渡金属催化剂催化，反应即可顺利进行.

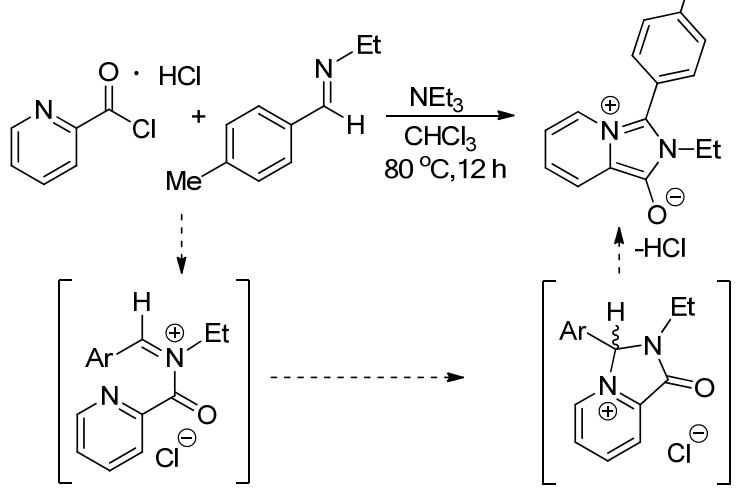

图式 28 以吡啶酰氯为原料合成唑类介离子合物

Scheme 28 Synthesis of azole mesoionic compounds using pyridine acyl chloride as raw material

2018 年, Samarskaya 等 ${ }^{[54]}$ 报道了盐酸亚硒酮在二氯 甲烷溶剂中，强碱的作用下，使盐酸亚硒酮去质子化， 与亲电试剂膦酸酯酰氯发生亲核加成消除反应，生成惡 二唑介离子化合物(Eq. 8). 此方法高效简便, 所需原料 只需在克级, 此反应便能顺利的完成, 且产率较高.

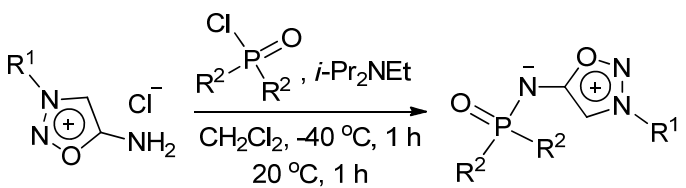

2018 年, Pearson 等 ${ }^{[55]}$ 报道了以扁桃酸为起始原料, 加入乙醇, 硫酸中加热生成酯. 在干燥的氯仿溶剂中, 利用三溴化磷对其溴化. 再在 1.1 equiv. 的 KSAc 下进行 亲核取代反应，生成了高产率的硫乙酰基中间体。随后 
用乙醇钠处理硫乙酰基获取硫醇, 经过酯基水解与颈基 亚硝化后, 在 1 equiv. 二环己基碳二亚胺(DCC)作用下 脱水闭环合成了介离子化合物(Scheme 29). 当 $\mathrm{R}^{1} 、 \mathrm{R}^{2}$ 、 $\mathrm{R}^{3} 、 \mathrm{R}^{4}=\mathrm{H}$ 时产率可达 $90 \%$ 左右.

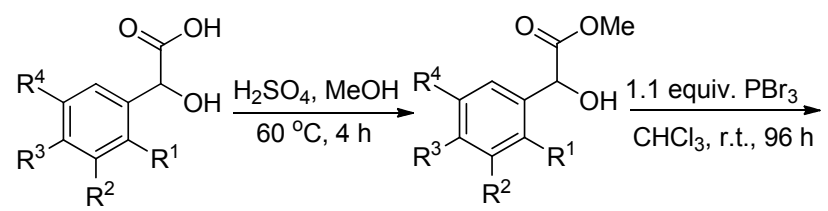

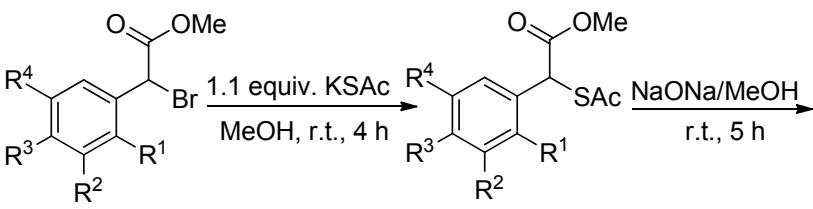

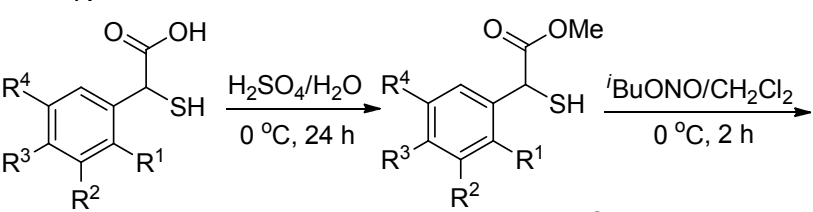

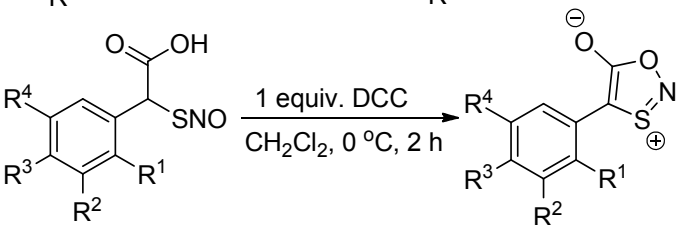

图式 29 噻异噁唑介离子化合物合成

Scheme 29 Synthesis of mesogenic compound

2019 年, 宋宝安等 ${ }^{[56]}$ 为了寻求具有较高杀虫和抗 菌活性的介离子类药物分子, 在前期研究基础上, 本工 作以商品药剂三氟苯嘧啶(TFM)为先导, 在保留三氟甲 基苯基的前提下, 将 5-氨基-1,3,4-噻二唑-2-硫醇结构与 丙二酸酯单元杂化构建 1,3,4-噻二唑并 [3,2- $\alpha$ 嘧啶母环; 同时, 以 dicloromezotiaz 的噻唑杂环代替 TFM 中 1 位 的嘧啶环, 设计合成了一系列 1,3,4-噻二唑并 [3,2- $\alpha$ ]嘧 啶类介离子化合物(Scheme 30). 并对化合物进行了杀 虫活性以及抑菌活性测试. 结果表明在浓度为 $100 \mu \mathrm{g} /$<smiles></smiles>

图式 30 1,3,4-噻二唑并 $[3,2-a]$ 嘧啶类介离子化合物 Scheme 30 Synthesis of 1,3,4-thiadiazolo[3,2-a]pyrimidine mesogenic compounds
$\mathrm{mL}$ 时，目标化合物对白背飞虫有一定的杀虫活性. 同 时在浓度为 $50 \mu \mathrm{g} / \mathrm{mL}$ 时, 部分化合物对水稻白叶枯病 菌，水稻细菌性条斑病菌和柑橘溃疡病菌表现出较好的 抑制作用.

2019 年, Kuzmina 等 ${ }^{[57]}$ 将 $N^{\prime}$-(二苯基亚甲基)乙酰肼 作为起始原料，在二甲基甲酰胺(DMF)溶剂中，在 $\mathrm{NaH}$ 作用下与 2-氯-5 氯甲基吡定发生亲核取代, 用盐酸处理 该化合物生成酰肼，对酰肼加热，使用 $\mathrm{P}_{2} \mathrm{~S}_{5}$ 进行硫化后 生成硫代酰肼, 在碱性条件下, 硫代酰肼与 $\mathrm{ClCO}_{2} \mathrm{CCl}_{3}$ 进行环化合成了含有噻二唑环的介离子化合物(Scheme 31).<smiles>CC(=O)NN=C(c1ccccc1)c1ccccc1</smiles><smiles>CC(=O)N(N)Cc1ccc(Cl)nc1</smiles>

图式 31 噻二唑介离子化合物的合成

Scheme 31 Synthesis of thiadiazole mesogenic compounds

2019 年, Benaissa 等 ${ }^{[58]}$ 将 5-溴咪唑并 $[1,5-\alpha]$ 溴化吡 啶鎓盐与丙二酸二甲酯或丙二酸二乙酯作为起始原料, 加入过量的碳酸钾, 在 $\mathrm{DMF}$ 溶剂和 $100{ }^{\circ} \mathrm{C}$ 下进行反应. 鎓盐首先在碱和有机溶剂的作用下，生成 $N$-杂环卡宾 (NHC)中间体，该中间体可作为 1,3-偶极子与丙二酸二 乙酯或丙二酸二甲酯亲核加成实现 $\mathrm{C}-\mathrm{C}$ 键的偶联，最 终环化脱去一分子 $\mathrm{HBr}$ 生成含有稠环的介离子类化合 (Scheme 32). 除了用作有机催化剂的 NHC，很少被视 为合成中潜在的中间体，在此反应中显示出高效的亲核 活性，通过分子内的环化，使得该反应简单易行，直接 高效, 为介离子化合物的合成提供了一种新的方法.

2020 年, Hansmann 等 ${ }^{[59]}$ 利用炔烃和 1,3-二氮杂-2氮杂苯丙烯盐进行环加成生成相应的甲基化 1,2,3-三唑 鎓盐，向鎓盐的 THF 溶液中加入 1 equiv.的三甲基甲硅 烷基酰胺钾后，1,2,3-三唑鎓盐上的甲基去质子化，使用 戊烷萃进行, 以深紫色固体形式分离出含有咪唑介离子 化合物(Scheme 33). 收率为 $67 \%$. 该化合物对氧气高度 敏感, 但在惰性气氛中可在数天内以固体或溶液形式稳 定.

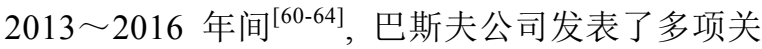
于介离子类化合物的专利. 2013 年发表专利中把六元介 

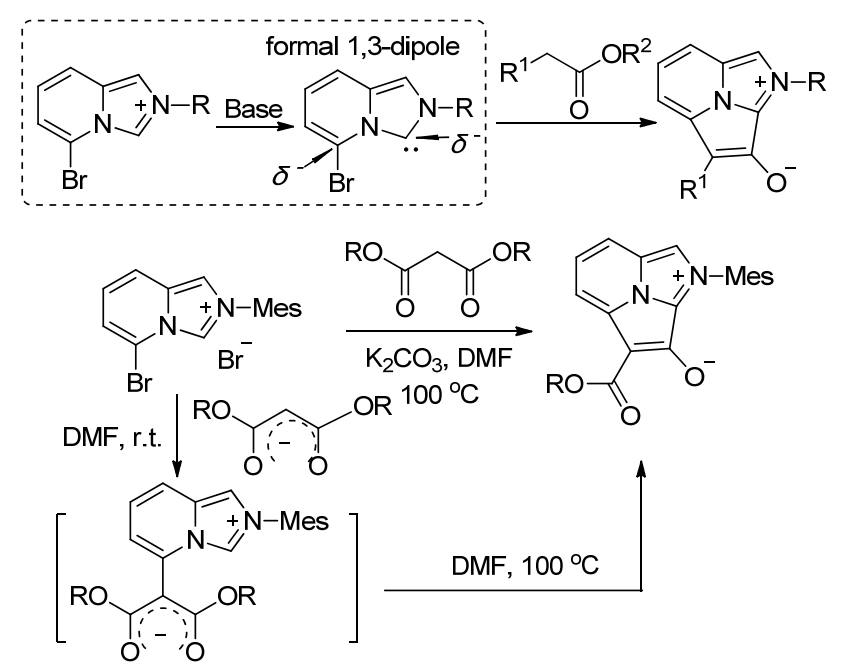

图式 32 含有稠环的介离子类化合物

Scheme 32 Synthesis of fused rings mesoionic compounds<smiles>CCC([N+](=O)[O-])C(F)(F)F</smiles><smiles>CCO[P+]([O-])c1c(C)nc(-c2ccccc2)n1-c1ccccc1</smiles>

图式 33 咪唑介离子化合物的合成

Scheme 33 Synthesis of Imidazole mesogenic compounds

离子核心环改为五元噻(噁)二唑环; 2014 年发表 3 篇专 利中分别在六元介离子嘧啶酮环 5 7 位引入各种杂环 或者苯环, 以及对 3-位和侧链进行衍生; 2016 年发表的 专利对六元介离子嘧啶酮环的侧链进行修饰. 但是这些 结构的修饰并没有提高杀虫活性, 只有少数化合物在浓 度为 $500 \mu \mathrm{g} / \mathrm{mL}$ 时对褐飞虫的防效超过 $75 \%$.<smiles></smiles>

2013<smiles></smiles>

2014<smiles></smiles>

2014

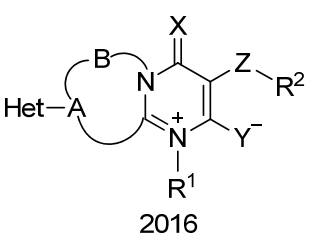

2017 年, 拜耳公司发表了一类卤代 3-(乙酰基)-1[(1,3-噻唑-5-基)甲基]-( $1 H$-咪唑并 $[1,2-\alpha]$ 吡啶-4-鎓 2-盐 类介离子衍生物 $\mathbf{1}$ 的专利 ${ }^{[65]}$, 并将其作为潜在的杀螨剂
和杀虫剂进行研究. 专利中报道的大多数化合物在浓度 为 $100 \mu \mathrm{g} / \mathrm{mL}$ 时对绿桃蚜的防效达 $100 \%$. 2018 年, 拜耳 公司又报道了一类咪唑并 [1,2- $\alpha$ ]吡啶介离子衍生物 $\mathbf{2}^{[66]}$, 其中化合物 2 对黄瓜条叶甲有 $100 \%$ 防效.<smiles></smiles><smiles></smiles>

2018 年, Holyoke ${ }^{[67]}$ 公开了一类五到七元环并咪唑 类的介离子化合物 3. 其中部分化合物在浓度为 $50 \mu \mathrm{g} /$ $\mathrm{mL}$ 时对马铃薯叶蝉和玉米灰飞闽的致死率均为 $80 \%$.

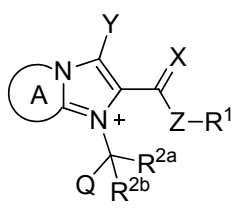

3

\section{2 总结与展望}

介离子化合物拥有广泛的生物活性及其重要的应 用价值，自人们首次发现悉尼酮后，随后的近百年来介 离子类化合物已取得长足的发展. 本文归纳总结了介离 子化合物的主要合成方法及应用, 但不同程度地存在着 诸多问题. 例如类型比较少、合成方法单一及稳定性差 等问题．随着对介离子认识的不断深入，以三氟苯嘧啶 为代表的一些介离子化合物作为农药已应用于农业生 产中. 因此该类化合物的合成及应用值得人们进一步去 探索.

\section{References}

[1] Earl, J. C.; Mackney, A. W. J. Chem. Soc. 1935, 899.

[2] Huisgen, R.; Gotthardt, H.; Bayer, H, O. Angew. Chem., Int. Ed. 1964, 3, 136

[3] St Cyr, D. J.; Arndtsen, B. A. J. Am. Chem. Soc. 2007, 129, 12366

[4] Wilton, D. J.; Harrison, R. F.; Willett, P.; Delaney, J.; Lawson, K.; Mullier, G. J. Chem. Inf. Model. 2006, 46, 471.

[5] Ollis, W. D.; Ramsden, C. A. Adv. Heterocycl. Chem. 1976, 19, 1.

[6] Latthe, P. R.; Shinge, P. S.; Badami, B. V.; Patil, P. B.; Holihosur, S. N. J. Chem. Sci. 2006, 118, 249.

[7] Gotthard, H.; Huisgen, R.; Schaefer, F. C. Tetrahedron Lett. 1964 10,487

[8] Zhang, W. M.; Holyoke, C. W.; Barry, J.; Leighty, R. M.; Cordova, D.; Vincent, D. R.; Briddell, T. A. Bioorg. Med. Chem. Lett. 2016, 26, 5444.

[9] Zhang, W. M. Acc. Chem. Res. 2017, 50, 2381

[10] Holyoke, C. W. Jr.; Cordova, D.; Zhang, W. M.; Barry, D. J.; Leighty, M. R.; Dietrich, F. R.; Rauh, J. J.; Pahutski, F. T. Jr.; Lahm, P. G.; Tong, T. M.-H.; Benner, A. E.; Andreassi, L. J.; Smith, M. R.; Vincent, R. D.; Christianson, A. L.; Teixeira, A. L.; Singh, V.; Hughes, A. K. Pest Manage. Sci. 2017, 73, 796

[11] Coburn, R. A.; Carapellotti, R. A. J. Pharm. Sci. 1976, 65, 1505.

[12] White, E. H.; Egger, N. J. Am. Chem. Soc. 1984, 106, 3701.

[13] Chandrasekhar, R.; Nanjan, M. J. Mini. Rev. Med. Chem. 2012, 12, 
1359

[14] Jogul, J. J.; Badami, B. V. J. Serb. Chem. Soc. 2006, 71, 851.

[15] Kennis, L. E. J.; Bischoff, F. P.; Mertens, C. J.; Love, C. J.; Keybus, F. A. F. V.; Pieters, S.; Braeken, M.; Megens, A. A. H. P.; Leysen, J. E. Bioorg. Med. Chem. Lett. 2000, 10, 71.

[16] Holyoke Jr, C. W.; Zhang, W. M.; Pahutski, T. F. Jr.; Lahm, G. P.; Tong, M. H. T.; Cordova, D.; Leighty, R. M. 13th IUPAC International Congress of Pesticide Chemistry - Discovery and Synthesis of Crop Protection Products, American Chemical Society, Washington, DC, USA, 2015, pp. 365 378 .

[17] Moustafa, M. A.; Gineinah, M. M.; Nasr, M. N.; Bayoumi, W. A. Arch. Pharm. Med. Chem. 2004, 337, 427.

[18] Latthe, P. R.; Shinge, P. S.; Badami, B. V.; Patil, P. B.; Holihosur, S. N. J. Chem. Sci. 2006, 118, 249.

[19] Taj, T.; Kamble, R. R.; Gireesh, T. M.; Hunnur, R. K. J. Serb. Chem. Soc. 2011, 76, 1069.

[20] Patel, K. C.; Patel, H. D. E.-J. Chem. 2011, 8, 113.

[21] Hegde, J. C.; Girish, K. S.; Adhikari, A.; Kalluraya, B. Synth. Commun. 2013, 43, 301

[22] Dubey, R.; Chaudhary, N.; Kumar, R.; Panwar, H. Orient. J. Chem. 2014, 30, 271

[23] Bayer, H. O.; Huisgen, R.; Knorr, R.; Schaefer, F. C. Chem. Ber. 1970, 103, 2581.

[24] Potts, K.; Yao, S. J. Org. Chem. 1979, 44, 977

[25] Anderson, A. K.; Heider, A. R. Synth. Commun. 1986, 16, 357.

[26] Kato, H.; Tani, K.; Kurumisawa, H.; Tamura, Y. Chem. Lett. 1980, 717.

[27] Lu, Y.; Arndtsen, B. A. Angew. Chem., Int. Ed. 2008, 47, 5430

[28] (a) Arndtsen, B. A. Chem.-Eur. J. 2009, 15, 302.

(b) Quesenel, J. S.; Amdtsen, B. A. Pure. Appl. Chem. 2013, 85, 377.

[29] Miao, Q.; Sun, H. L. Chin. Sci. Bull. 2015, 60, 2003 (in Chinese). (苗群，孙怀林，科学通报，2015, 60, 2003.)

[30] Miao, Q. M.S. Thesis, Nankai University, Tianjin, 2015 (in Chinese). (苗群，硕士论文，南开大学，天津, 2015.)

[31] Torres, G. M.; Quesnel, J. S.; Bijou, D.; Arndtsen, B. A. J. Am. Chem. Soc. 2016, 138, 7315 .

[32] St Cyr, D. J.; Arndtsen, B. A. J. Am. Chem. Soc. 2007, 129, 12366.

[33] Couture, A.; Deniau, E.; Grandclaudon, P. Synthesis 1994, 953.

[34] Morin, M. S. T.; St Cyr, D. J.; Arndtsen, B. A. Org. Lett. 2010, 12 , 4916.

[35] Morin, M. S. T.; Aly, S.; Arndtsen, B. A. Chem Commun. 2013, 49, 883.

[36] Jeanmart, S.; Edmunds, A. F.; Lamberth, C. Bioorg. Med. Chem. 2016, 24,317

[37] Holyoke, C. W.; Tong, M. T.; Coats, R. A. WO 099929, 2009.

[38] Lugan, N.; Lavigne, G.; Labande, A.; Cesar, V. Organometallics 2014, 33, 5085 .

[39] Zhang, W. M.; Holyoke, W. C. Jr.; Pahutski, F. T. Jr.; Lahm, P. G.; Barry, D. J.; Cordova, D.; Leighty, M. R.; Singh, V.; Vincent, R. D.; Tong, T. M.-H.; Hughes, A. K.; Mc Cann, F. S.; Henry, T. Y.; Xu, M.; Briddell, A. T. Bioorg. Med. Chem. Lett. 2017, 27, 16.

[40] Lei, G. Y.; Ying, J. W.; Song, Y. Q. Mod. Agrochem. 2017, 16, 14 (in Chinese).

(雷月光, 英君伍, 宋玉泉, 现代农药, 2017, 16, 14.)

[41] Zhang, W. M.; Holyoke, W. C. Jr.; Barry, J.; Cordova, D.; Leighty, M. R.; Tong, T. M.-H.; Hughes, A. K.; Lahm, P. G.; Pahutski, F. T. Jr.; Xu, M.; Briddell, A. T.; Mc Cann, F. T.; Henry, T. Y.; Chen, Y. Z. Bioorg. Med. Chem. Lett. 2017, 27, 911.

[42] (a) Liu, D. Y.; Zhang, J.; Zhao, .L; He, W. J.; Liu, Z. J.; Gan, X. H.; Song, B. A. J. Agric. Food Chem. 2019, 67, 11860

(b) Pan, J. K. M.S. Thesis, Guizhou University, Guiyang, 2017 (in
Chinese).

(潘建科, 硕士论文, 贵州大学, 贵阳, 2017.)

[43] Hasegawa, S.; Kamo, T.; Kagohara, Y.; Miyake, T.; Kobayashi, T.; Matsuda, R.; Asano, S.; Kudamatsu, A. WO 171053, 2016.

[44] Thieme, P.; Patsch, M.; Konig, H. Liebigs Ann. Chem. 1972, 764, 94.

[45] Zhang, Z. Y.; Zhu, C. H.; Sun, X. W. Chem. J. Chin. Univ. 2000, 21,386 (in Chinese).

(张自义, 褚长虎, 孙小文，高等学校化学学报, 2000, 21, 386.)

[46] Abbott, P. A.; Bonnert, R. V.; Caffrey, M. V.; Cage, P. A.; Cooke, A. J.; Furber, M.; Hill, S.; Withnall, J. Tetrahedron 2002, 58, 3185.

[47] Avalos, M.; Babiano, R.; Cabanillas, A.; Cintas, P.; Higes, F. J.; Jiménez, J. L.; Palacios, J. C. J. Org. Chem. 1996, 61, 3738.

[48] Arévalo, M. J.; Avalos, M.; Babiano, R.; Cintas, P.; Hursthouse, M. B.; Jiménez, J. L.; Light, M. E.; Palacios, J. C. Tetrahedron: Asymmetry 2002, 13, 223.

[49] Avalos, M.; Babiano, R.; Cintas, P; Jime'nez, J. L.; Palacios, J. C. Acc. Chem. Res. 2005, 38, 460 .

[50] Chernyshev, V. N.; Astakhov, A. V.; Starikova, Z. A. Tetrahedron 2010, 66, 3301.

[51] Hassan, A. A.; Bräse. S.; Tawfeek, H. N.; Mohamed, N. K.; Nieger, M.; El-Shaieb, K. M. A. Tetrahedron Lett. 2014, 55, 2385.

[52] Zhao, S. X.; Yu, R. C.; Chen, W. Z.; Liu, M. C.; Wu, H. Y. Org. Lett. 2015, 17, 2828

[53] Erguven, H.; Leitch, D. C.; Keyzer, E. N.; Arndtsen, B. A. Angew. Chem., Int. Ed. 2017, 56, 6078 .

[54] Samarskaya, A. S.; Cherepanov, I. A.; Godovikov, I. A.; Dmitrienko, A. O.; Moiseev, S. K.; Kalinin, V. N.; Hey-Hawkins, E. Tetrahedron 2018, 74, 2693.

[55] Eilertsen, M.; Allin, S. M.; Pearson, R. J. Bioorg. Med. Chem. Lett. 2018, 28, 1106.

[56] (a) He, W. J.; Liu, D. Y.; Gan, X. H.; Zhang, J.; Liu, Z. J.; Yi, C. F.; Song, B. A. Chin. J. Org. Chem. 2019, 39, 2287 (in Chinese) (何文静, 刘登曰, 甘秀海, 张建, 刘峥军, 易崇粉, 宋宝安, 有 机化学, 2019, 39, 2287.)

(b) He, W. J. M.S. Thesis, Guizhou University, Guiyang, 2019 (in Chinese).

(何文静，硕士论文，贵州大学，贵阳, 2019.)

[57] Kuzmina, O. M.; Weisel, M.; Narine, A. A. Eur. J. Org. Chem. 2019, 5527.

[58] Benaissa, A.; Pallova, L.; Morantin, M. E.; Lafitte, T.; Huynh, M.; Barthes, C.; Vendier, L.; Lugan, N.; Bastin, S.; César, V. Chem.-Eur. J. 2019, 3, 242.

[59] Hansmann, M. M.; Antoni, P. W.; Pescht, H. Angew. Chem., Int. Ed. 2020, $59,2$.

[60] Narine, A.; Dickhaut, J.; Kaiser, F.; Bandur, N. G.; Koerber, K.; Von Deyn, W.; Derksen, S.; Paulini, R.; Culbertson, D. L. WO 144228, 2013.

[61] Dickhaut, J.; Narine, A.; Derksen, S.; Bandur, N. G.; Von Deyn, W.; Koller, R.; Wach, J. Y.; Langewald, J.; Rankl, N. B. WO 202582, 2014.

[62] Narine, A.; Bandur, N. G.; Dickhaut, J.; Derksen, S.; Koller, R.; Von Deyn, W.; Wach, J.-Y.; Culbertson, D. L. WO 167084, 2014.

[63] Narine, A.; Dickhaut, J.; Kaiser, F.; Bandur, N. G.; Koerber, K.; Von Deyn, W. WO 033244, 2014

[64] Dickhaut, J.; Narine, A.; Von Deyn, W.; Koller, R.; Wach, J.-Y.; Vyas, D.; Adisechan, A.; Shinde, H. WO 055431, 2016.

[65] Markus, H.; Daniela, P.; Kerstin, I.; Ulrich, G.; Silvia, C. G.; Marc, M.; Sascha, E.; Andreas, T. WO 093214, 2018.

[66] Heil, M.; Hoffmeister, L.; Webber, M.; Ilg, K.; Goergens, U.; Turberg, A. WO 192872, 2018

[67] Holyoke, W. C. Jr. WO 208595, 2018 